\title{
Novel Fuzzy Filters for Noise Suppression from Digital Grey and Color Images
}

\author{
Geeta Hanji \\ Faculty \\ PDACE, Gulbarga
}

\author{
Basaveshwari C. \\ Student \\ PDACE, Gulbarga, India
}

\author{
M.V. Latte \\ Principal \\ JSSAT, Bangalore, India
}

\begin{abstract}
Images are rich information carriers and (such as medical images) are normally contaminated by additive and substitutive noise which makes the extraction of features (and clinical data analysis) difficult. Hence to enhance the image quality prior to post processing, image pre-processing operations such as de-noising with linear and non-linear filters have been applied traditionally. Recently nonlinear filtering techniques have been assumed a lot of significance as they are capable of suppressing the effects of substitutive (salt and pepper impulsive noise of low to high noise levels) and additive (Gaussian noise of low to medium noise levels) noise types and to preserve the important signal/image details such as edges and fine details and suppress the degradations occurring at the time of image/signal formation or transmission through nonlinear channels, during storage and retrieval. Broadly speaking, image filters exist in transform and spatial domains. Spatial domain nonlinear filters are more versatile than their counterparts, namely linear filters. Spatial domain nonlinear fuzzy classical filters are simply modification/extension of the classical median and moving average filtering approaches, offer several advantages over classical nonlinear filters, and using simple fuzzy rules it is easy to realize them. They are also capable of reasoning with vague and uncertain information. Work presented in this paper deals with nonlinear median based and linear average based fuzzy filters and aims at fulfilling three objectives, viz; (i) To systematically study the performance of classical nonlinear median and fuzzy median and average filters for the removal of impulse and Gaussian noise from gray and color images that have been corrupted from low to high values of noise and to present an experimental review to identify the best algorithm within the frame work of classical fuzzy median filters. (ii)To propose : (a) an impulse classifier based fuzzy switching median filter and (b) the design of a multi pass cascaded fuzzy filter for noise cancellation, and explore their applications to reduce noise in images with random and impulse characteristics. Finally to conclude the work a comparative study is done and the computational aspects are analyzed with the help of mean square error (MSE), peak signal to noise ratio (PSNR), and 2D correlation (COR) and some future solutions are proposed.
\end{abstract}

\section{Keywords}

Fuzzy filter, Impulse noise, Gaussian Noise, Image Processing, Membership Function, Median filter, Cascaded filter, Noise Suppression.

\section{INTRODUCTION}

Images are rich in information and are often contaminated with the principal noise sources affecting during the process of capture (digitization) and/or transmission/ storage/ retriviation. A variety of factors, such as environmental conditions during the process of image acquisition and the quality of the sensing elements themselves are the major cause for image degradations due to noise effects. Apart from this, the digital images are often contaminated during transmission principally due to the transmission channel interference/impairments/noise. Noise in images is generally undesirable, disturbing and not only degrades the visual resolution but also impedes the quantitative analysis of an image, as it always plays a negative role on higher level processing tasks such as classification, and segmentation. Thus, for better understanding and interpretation of digital images, de-noising becomes a fundamental and a preliminary step to suppress the negative effects of noise from the corrupted images and preferably keep the structures of the original image simultaneously [1] and prepare it ready for further processing such as classification, segmentation, encoding, recognition, compression, tracking, and texture analysis [2]. In other words, without filtering as a preprocessing operation, further processing of digital images would have presented inappropriate results. Impulse (salt and pepper) and Gaussian (random) noise are two common noise types in digital images that can be expressed in terms of noise density and mean/variance respectively. Reducing the effects of random noise is a more challenging task than the impulsive noise. In the field of image processing, a vast number of spatial domain linear and nonlinear filters have been proposed for image de-noising applications. Linear filters possess the mathematical simplicity, and effective suppression of noise with spectral components. However, the main drawback of linear filters is their inability to suppress the effects of impulsive noise with good edge preservation.

Nonlinear spatial domain noise-reduction algorithms, ranging from a simple median filtration to the regularization approaches have been proved efficient in image noise suppression with details preserved intact, as they are capable of exploiting the spatial order of the surrounding pixels together with rank order. Since the introduction of median filter in early 1970 'by Tukey [3], the popularity of spatial domain nonlinear median filter and its several variants have been continuously increasing and gained a lot of significance since the last couple of years. The basic idea behind median filter is to replace the central pixel in the current window by the median of sorted array of the current window pixel. Median filter is regarded as a special case of non-linear filters used for smoothing signals while reducing the image noise and has been widely employed in suppressing image noise and proven to be a successful and outperforming many conventional de-noising filters due to their special and preferred properties [1-3] namely, it's effectiveness in diminishing the effects of substitutive noise such as a fixed value impulsive (or salt and pepper) noise without distorting the minute details of the information rich images such as edges, fine lines [4] and generally known to be a much better alternative and option than any linear filter at suppressing additive noise (AWGN) effects of medium level with edge preservation. However, for higher levels of additive noise it is debatable if this is the case or not [4], and in either case it is 
not particularly quite effective when compared to improved and more advanced techniques. Deficiencies of a median (MED) filter [5-7] in suppressing non-impulsive noise can be overcome by some success by another non-linear filtering process, namely moving average (MAV) filter. The basic idea behind a standard moving average filter is to substitute for its center pixel value, an average pixel value of its pre-defined neighbors. However, suppressing the effects of both the Gaussian and impulsive noise effects with these filters is a non-trivial task. In this regard, median and moving average filtering using fuzzy concepts have been developed in the past [5].Nonlinear filters such as fuzzy classical filters are simple as they are extension /modification of basic classical median and moving average filters. Generally speaking, classical fuzzy median and moving average filter [6-7] techniques with different membership functions are nonlinear, knowledgebased, and are able to process the imperfect data if this imperfection begins from vagueness and ambiguity rather than randomness. An applied review of different fuzzy filters and results of a broad comparison study have been made available in [8]. Authors state that, median and average filtering using fuzzy concepts described in [5-6]use Gaussian and symmetrical/asymmetrical triangular membership functions have been demonstrated varied success in suppressing the effects of impulsive and random noise. Different types of new hybrid filtering techniques for the removal of Gaussian noise from ultrasound medical images, center weighted hybrid filtering techniques and also fuzzy hybrid filtering techniques [9-11] for simultaneous removal of Gaussian and speckle noises have been also described. The fuzzy median filter [12-13], is a modification to the classical median filter [12-13] and aims at low to medium amount of image noise suppression from the digital images. Work presented through this paper aims at fulfilling three objectives, viz; (i) To systematically study the performance of classical nonlinear median and fuzzy median filters for the removal of impulse and Gaussian noise from gray and color images that have been corrupted from low to high values of noise and to present an experimental review to identify the best algorithm within the frame work of classical fuzzy median filters. To propose (ii) an impulse classifier based fuzzy switching median filter and (iii) the design of a multi pass cascaded fuzzy filter for noise cancellation, and explore their applications to reduce the noise effects in images with random and impulse characteristics. Proposed work is organized as follows.

A review of classical median and fuzzy filters is presented in section 2. Section 3 deals with the proposed fuzzy switching median filter and a multi-pass cascaded fuzzy filtering techniques for de-noising the standard and medical images. Both quantitative and qualitative results, comparisons and discussions have been provided in section 4 , followed by the conclusions and scope for further work in Section 5.

\section{A REVIEW OF FUZZY MEDIAN AND CLASSICAL MEDIAN FILTERS}

Since, the proposed work aims at developing a 2-stage (or switching) fuzzy filter, and a multi pass cascaded fuzzy filter for reducing the effects both impulsive and Gaussian noise from the noisy digital images, and comparing the de-noising results obtained with the basic median filter and fuzzy median filters, a brief review [5-8] of the classical median and fuzzy median filters has been presented in this section. In general, the output of the filter is defined as

$y(i, j)=\frac{\sum_{r, s \in W} F[X(i+r, j+s)] \cdot X(i+r, j+s)}{\sum_{r, s \in W} F[X(i+r, j+s)]}$

where $X(i+r, j+s)$ is input image, $F[X(i+r, j+s)]$ is the general window function and $\mathrm{W}$ is the size (or area) of the window. For a square window of dimensions $\mathrm{N} \times \mathrm{N}$, the range of ' $\mathrm{r}$ ' and ' $\mathrm{s}$ ' are $-\mathrm{R} \leq \mathrm{r} \leq \mathrm{R}$ and $-\mathrm{S} \leq \mathrm{s} \leq \mathrm{S}$, where $\mathrm{N}=2 \mathrm{R}+1$ $=2 \mathrm{~S}+1$.Various fuzzy filters are obtained with different definitions of the window functions (or different weighted membership functions) such as asymmetrical triangular fuzzy filter with median center (ATMED), with moving average center (ATMAV), symmetrical triangular fuzzy filter with median center (TMED), with moving average center (TMAV), Gaussian fuzzy filter with median center (GMED) and with moving average center (GMAV). Figure (1) represents the general block diagram of fuzzy median and average filters.

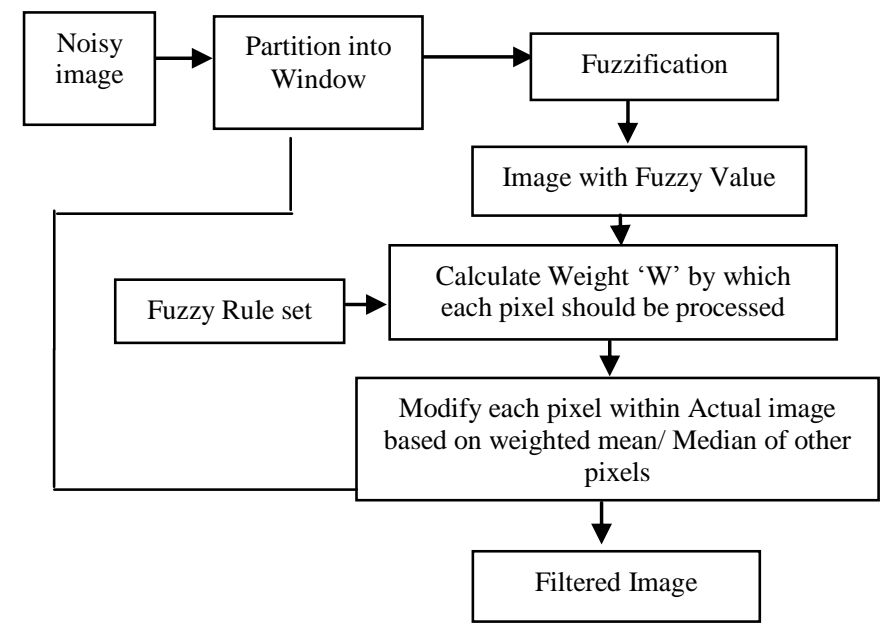

Fig 1: General Block Diagram of Classical Fuzzy Filters 
Mentioned below are the definitions of a moving average (MAV), median filter (MED), ATMED, ATMAV and GMED filters.

\subsection{MAV}

The window function in case of moving average filter is given as follows.

$$
F\left[X(i+r, j+s)=\left\{\begin{array}{lr}
1 ; & \text { for } X(i+r, j+s)=X_{\text {mav }}(i, j) . \\
0 ; & \text { otherwise }
\end{array}\right.\right.
$$

The output value $\mathrm{Y}(\mathrm{i}, \mathrm{j})$ at the center of window $\mathrm{W}$ is substituted by the average pixel intensity $X_{\text {mav }}(i, j)$ among the input pixel intensities $\mathrm{X}(\mathrm{i}+\mathrm{r}, \mathrm{j}+\mathrm{s})$ for $\mathrm{r}, \mathrm{s} \in \mathrm{W}$ at discrete indices $(\mathrm{i}, \mathrm{j})$.

\subsection{MED}

The window function in case of a standard median filter is given as follows.

$F[X(i+r, j+s)]=\left\{\begin{array}{lr}1 ; \text { for } X(i+r, j+s)=X_{\text {med }}(i, j) . \\ 0 ; & \text { otherwise }\end{array}\right.$

The output value $\mathrm{Y}(\mathrm{i}, \mathrm{j})$ at the center of window $\mathrm{W}$ is substituted by the median pixel intensity $X_{\text {med }}(i, j)$ among the input pixel intensities $X(i+r, j+s)$ for $r, s \in W$ at discrete indices $(i, j)$

\subsection{Asymmetrical Triangular Fuzzy Filter with Median Center (ATMED)}

Basic ATMED filter is a single stage filter without noise classifier [70].This filter is defined by applying asymmetrical triangular fuzzy membership weighted function expressed in equation (1) to the image pixel values within a moving window.

$$
\begin{gathered}
F[X(i+r, j+s)] \\
=\left\{\begin{array}{c}
1-\frac{\left|\left[X_{\text {med }}(i, j)-X(i+r, j+s)\right]\right|}{\left|\left[X_{\text {med }}(i, j)-X_{\min }(i, j)\right]\right|}, \text { if } X_{\text {min }}(i, j) \leq X(i+r, j+s) \leq X_{\text {med }}(i, j) . \\
1-\frac{\left|\left[X X(i+r, j+s)-X_{\text {med }}(i, j)\right]\right|}{\left|\left[X_{\text {max }}(i, j)-X_{\text {med }}(i, j)\right]\right|}, \text { if } X_{\text {med }}(i, j) \leq X(i+r, j+s) \leq X_{\text {max }}(i, j) \\
1, \quad \text { if } X_{\text {med }}(i, j)-X_{\text {min }}(i, j)=0 \text { or } X_{\text {max }}(i, j)-X_{\text {med }}=0
\end{array}\right.
\end{gathered}
$$

Where $X_{\min }(i, j), X_{\text {med }}(i, j), X_{\max }(i, j)$ are the minimum , median and maximum values within the window at discrete index (i, $j)$. The degree of asymmetry depends on the difference between $X_{\text {med }}(i, j)-X_{\min }(i, j)$ and $X_{\max }(i, j)-X_{\text {med }}(i, j)$.

\subsection{ATMAV}

Filter with asymmetrical triangular fuzzy membership function with the moving average as its center within a working window is defined as follows.

$$
\begin{aligned}
& F[X(i+r, j+s)] \\
& =\left\{\begin{array}{c}
1-\frac{\left|\left[X_{\text {mav }}(i, j)-X(i+r, j+s)\right]\right|}{\left|\left[X_{\operatorname{mav}}(i, j)-X_{\min }(i, j)\right]\right|}, \text { if } X_{\min }(i, j) \leq X(i+r, j+s) \leq X_{\text {mav }}(i, j) . \\
1-\frac{\mid\left[\left[X(i+r, j+s)-X_{\text {mav }}(i, j)\right] \mid\right.}{\left|\left[X_{\max }(i, j)-X_{\operatorname{mav}}(i, j)\right]\right|}, \text { if } X_{\text {mav }}(i, j) \leq X(i+r, j+s) \leq X_{\max }(i, j) \\
1, \quad \text { if } X_{\text {mav }}(i, j)-X_{\text {min }}(i, j)=0 \text { or } X_{\max }(i, j)-X_{\text {mav }}=0
\end{array}\right.
\end{aligned}
$$

\subsection{GMED}

Filter with Gaussian fuzzy membership function with the median as its center within a working window is defined as follows.

$F[X(i+r, j+s)]=e^{-1 / 2}\left[\frac{\left[x(i+r, j+s)-x_{\text {med }}(i, j)\right.}{\sigma(i, j)}\right] ;$ for $(r, s) \in A$

In the above equation, $\mathrm{X}_{\max }(\mathrm{i}, \mathrm{j}), \mathrm{X}_{\min }(\mathrm{i}, \mathrm{j})$ and $\mathrm{X}_{\operatorname{med}}(\mathrm{i}, \mathrm{j})$ are the maximum, minimum and the median values of all the input image pixel values $X(i+r, j+s)$ for all the values of $r, s \in$ $\mathrm{W}$ within the working window ' $\mathrm{W}$ ' at discrete indices ( $\mathrm{i}, \mathrm{j})$.

\subsection{TMED}

Triangular Fuzzy filter with the symmetrical triangular membership function with the window median value chosen at the center is defined as follows.

$$
\begin{gathered}
F[X(i+r, j+s)]=\left\{\begin{array}{c}
1-\frac{\mid X(i+r, j+s)-X_{\text {med }}(i, j)}{X_{P Q}(i, j)} \\
1 \quad ; \text { for }_{P Q}=0
\end{array} ;\right. \text { for } \\
\mid X(i+r, j+s)-X_{\text {med }}(i, j) \leq X_{Q}
\end{gathered}
$$

where $X_{P Q}(i, j)=\operatorname{Max}\left[X_{\max }(i, j)-X_{\text {med }}(i, j), X_{\text {med }}(i, j)-X_{\text {min }}(i, j)\right.$.

\section{DESCRIPTION OF THE PROPOSED} FILTERS

In this section, a two- stage classical median based ATMED filter by the name 'Fuzzy Switching Median Filter (FSMF)' and a cascaded (of ATMED and ATMAV) fuzzy median filter with multi-pass, by the name 'Multi-pass Cascaded Fuzzy Filter (MCFF)'structures are presented. Both the filter structures are able to overcome the deficiencies of basic and individual fuzzy filters efficiently in suppressing the impulse and Gaussian noise effects of higher levels with better preservation of the image details intact. Details of these filter structure are as follows.

\subsection{Proposed Switching ATMED or Fuzzy Switching Median Filter (FSMF)}

As already mentioned, the traditional and the simplest way to remove salt-and-pepper noise is by windowing the noisy image with a conventional median filter [2]. Median filter, the most popular nonlinear filter, is extensively applied to eliminate salt and pepper noise due to its outstanding computational efficiency. Since the discovery of the Standard Median Filter (SMF) by Tukey [4] who applied it to the smoothing of statistical data, filters of this class have been subject to growing interest. Pratt [3] was the first to use median filters in image processing. Median filter, its modifications, and the classical median based fuzzy filters [57] are generally implemented to all pixels in an image. Hence the noiseless pixels also get affected. As a result, their effectiveness in noise suppression is often at the expense of blurred and distorted image features and they effective only at low noise densities. A better way to circumvent this drawback is to incorporate some decision-making process to discriminate between uncorrupted and corrupted pixels. With this notion, an attempt is made to improve the noise suppression capabilities of the classical median based fuzzy filter, namely Asymmetrical Triangular membership based Median filter (ATMED). Proposed extension of ATMED filter is a simple two stage filter by the name Fuzzy Switching Median Filter, FSMF to detect and then correct the fixed value (salt and pepper) impulses. A simple min-max noise 
detection strategy is used for impulse detection and classification in the proposed approach. In the correction stage, only the noisy pixels are replaced by a normalized sum of weighted input pixels within a $3 \times 3$ filtering window given by equation (1). Variable window sizes used for noise detection (obtained based on experimentations) are shown in Table (1). For noise filtering, fixed $3 \times 3$ window is used.

Table 1.Suggested window size for noise detection

\begin{tabular}{|c|c|c|c|}
\hline$\%$ Noise density $\mathrm{D}$ & $\mathrm{D}<30 \%$ & $30 \leq \mathrm{D} \leq 50$ & $\mathrm{D}>50$ \\
\hline Window size $(\mathrm{N} \times \mathrm{N})$ & $3 \times 3$ & $5 \times 5$ & $7 \times 7$ \\
\hline
\end{tabular}

$\underset{\text { Noisy image }}{\text { Noise Detection }} \rightarrow$\begin{tabular}{c|} 
ATMED Filtering \\
De-noised image
\end{tabular}

Fig 2: Switching ATMED Filter

The working procedure for the proposed algorithm is as below.

Step1) Select the working window $\mathrm{W}_{\mathrm{i}, \mathrm{j}}$ of appropriate size as given in Table (1)

Step 2) Classify the pixels into noisy or noise free as follows.

$$
\text { IF } 0<X(i, j)<255
$$$$
\text { GO TO step } 3
$$

$$
\text { ELSE GO TO step } 4 .
$$

Step 3) No Filtering

$$
O(i, j)=X(i, j)
$$

\section{EXIT}

Step 4) Sort the pixels in the window as a vector and compute the minimum $\left(\mathrm{X}_{\min }\right)$, maximum $\left(\mathrm{X}_{\max }\right)$ and the median $\left(\mathrm{X}_{\text {meed }}\right)$ in the sorted array.

Step 5) IF $X_{\min } \leq X(i+r, j+s) \leq X_{\text {med }}(i, j)$,

THEN window function is given by,

$$
F[X(i+r, j+s)]=1 \frac{\mid\left(X_{\text {med }}(i, j)-X(i+r, j+s) \mid\right.}{X_{\text {med }}(i, j)-X_{\min }(i, j)}
$$

\section{ELSE GOTO step 6}

Step 6) $\operatorname{IF} X(i, j) \leq X(i+r, j+s) \leq X_{\max }(i, j)$

THEN the window function is given by,

$$
F[X(i+r, j+s)]=1-\frac{\left|\left[X(i+r, j+s)-X_{\text {med }}(i, j)\right]\right|}{\left|\left[X_{\max }(i, j)-X_{\operatorname{med}}(i, j)\right]\right|}(9)
$$

\section{ELSE GOTO step 7.}

$$
\text { Step 7) IF } X_{\text {med }}(i, j)-X_{\min }(i, j)=0 \text { or } X_{\text {max }}(i, j)-X_{\text {med }}(i, j)
$$

THEN window function is given by,

$$
F[X(i+r, j+s)]=1
$$

Step 8) Noisy pixel at the location (i, j) is replaced by the normalized sum of weighted input pixels within the window as given by Eq. (1).

\subsection{Proposed Multi-pass Cascaded Fuzzy (ATMED-ATMAV) Filter, (MCFF)}

In this section, we present a novel technique of filtering color and grey images affected with impulsive and Gaussian noise of varying noise densities and variances using a cascade of weak and single stage fuzzy filters namely, ATMED and ATMAV filters. A series of these two basic filtering stages in the processing chain (where each filter operates the next in turn) with an iterative implementation ensures the noise reduction from the noisy digital images to a large extent. Proposed filter is a novel Multi-pass Cascaded Fuzzy filter and is able to address the limitations of single stage filters, and is extremely good in suppressing the negative effects of Gaussian noise affected (medical) images. Figure (3) shows the block diagram of the proposed multi pass cascaded fuzzy filters.

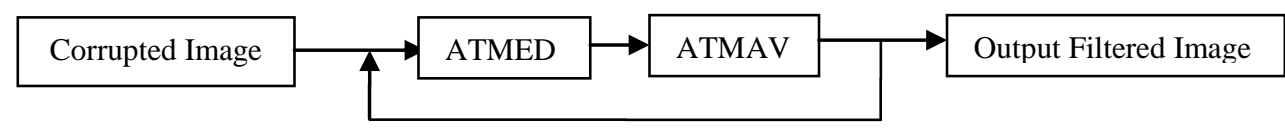

Fig 3: Block diagram of the proposed Multi-pass Cascaded Fuzzy (ATMED-ATMAV) Filter (MCFF).

\section{RESULTS AND DISCUSSIONS}

In this section, the experimental results performed on various standard test images such as Lena (Grey $(512 \times 512)$ and Color $(256 \times 256))$, Cameraman $(256 \times 256)$, Baboon $(256 \times$ $256)$, and few medical images such as Brain tumor $(256 \times$ $256)$, CT Head image $(256 \times 256)$ are presented. Before performing the image de-noising, all the test images were corrupted with fixed percentages of impulse noise and Gaussian noise. In these experiments, we added $10 \%-90 \%$ impulse noise and zero mean random noise at variance 0.01 to 0.09. The objective quantitative measures used for comparison are the Mean Square Error (MSE) between the original and reconstructed images, Peak Signal to Noise Ratio
(PSNR), Mean Absolute Error (MAE), and Correlation Coefficient (CC) defined as follows.

- Mean Square Error (MSE)

$$
\operatorname{MSE}=\frac{1}{M \times N} \sum_{i=0}^{M-1} \sum_{j=0}^{N-1}[O(i, j)-Y(i, j)]^{2}
$$

where $\mathrm{O}[\mathrm{i}, \mathrm{j}]$ is the original image and $\mathrm{Y}[\mathrm{i}, \mathrm{j}]$ is the de-noised image, $\mathrm{M} \times \mathrm{N}$ is the size of the image under consideration. 
- Peak Signal to Noise Ratio (PSNR)

$$
\text { PSNR }=\operatorname{10l}_{10} \frac{255^{2}}{\text { MSE }}
$$

For subjective evaluation, the quality of de-noised images and their edge details have been presented and analyzed.
De-noising results in terms of MSE and PSNR for various grey and color images are presented in the form of tables. The de-noising image results are presented for different images at different noise conditions. The edge retention ability of the proposed filter algorithms for the de-noised medical images are also presented to validate their edge preserving de-noising ability of the proposed filters in comparison with the other basic and state-of-art filters. Correlation Co-efficient (CC) results for the Cameraman images are also presented in the graphical form.

Table 2.Filtering performance of various filters in terms MSE for Cameraman image

\begin{tabular}{|c|c|c|c|c|c|c|c|c|}
\hline \% ND & MED & MAV & TMED & GMED & ATMED & ATMAV & FSMF (PA-I) & MCFF (PA-II) \\
\hline 10 & 63.25 & 708.4 & 317 & 217.2 & 256.6 & 531.1 & 32.09 & 3166 \\
\hline 20 & 130.3 & 994.6 & 330 & 266.5 & 273.8 & 375.6 & 72.74 & 2055 \\
\hline 30 & 356.5 & 1320.4 & 545 & 505.1 & 420.9 & 395.1 & 242.6 & 1359 \\
\hline 40 & 961.7 & 1726 & 1167 & 1158 & 946.1 & 537.3 & 614.8 & 891 \\
\hline 50 & 2104 & 6750 & 2249 & 2536 & 2186 & 784.6 & 1530 & 667 \\
\hline
\end{tabular}

MSE results presented in Table (2) show the efficacy of both the proposed filters in de-noising the low detail images such as Cameraman. Proposed filter-I, FSMF performs excellent up to $40 \%$ SPN compared to all the filters used for comparative analysis. However its filtering efficacy reduces drastically above $40 \%$. In contrast to the filtering performance of the proposed FSMF and other filters, proposed filter-II, MCFF exhibits very good filtering performance at higher noise situations and is quite obvious, since it uses a multi-pass and a cascaded structure.

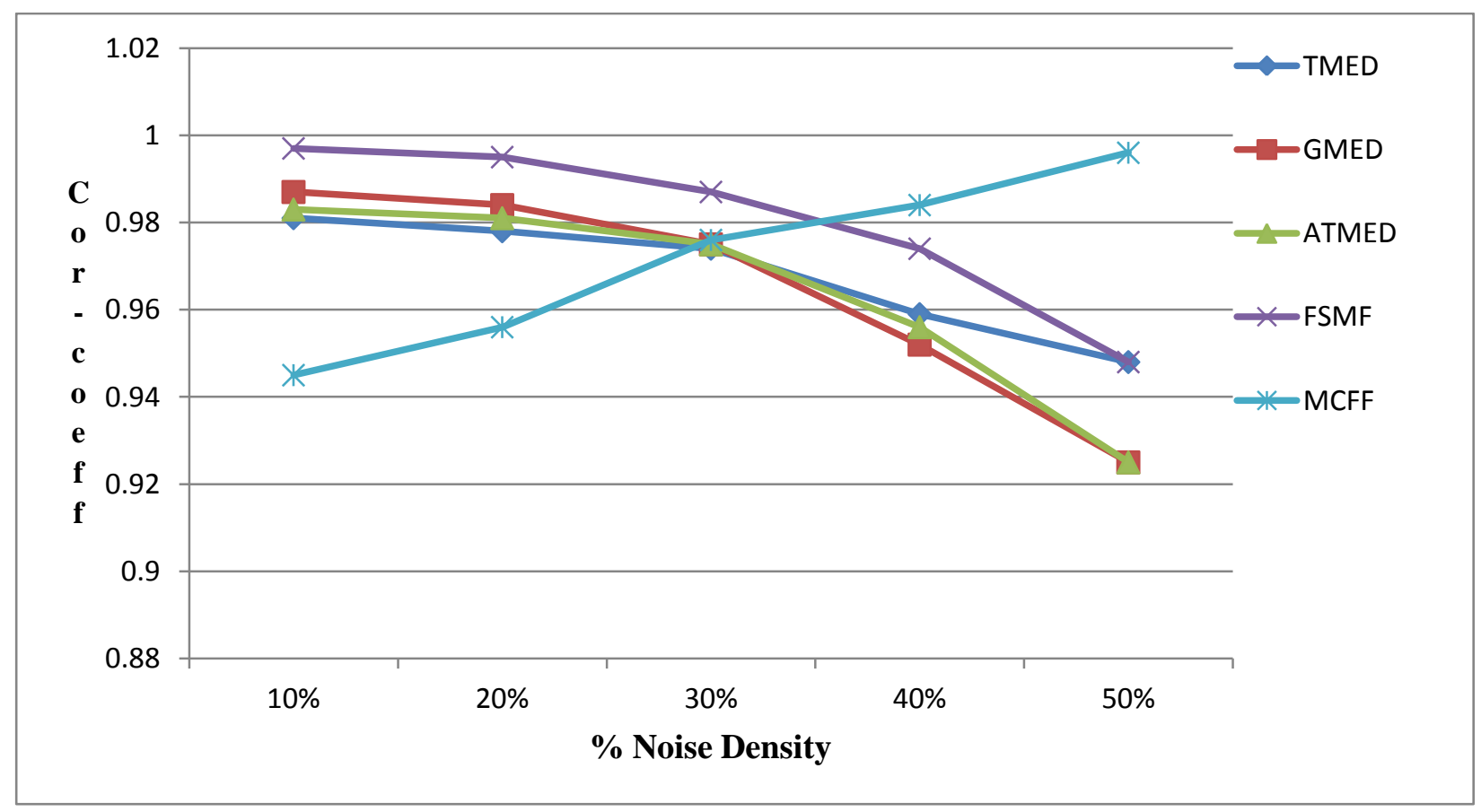

Fig 4: Correlation Co-efficient (CC) performance comparison of basic median, fuzzy and the proposed filters 
Table 3.PSNR results obtained for basic fuzzy and proposed fuzzy filters for different images contaminated with SPN.

\begin{tabular}{|c|c|c|c|c|c|c|c|c|c|c|c|c|c|}
\hline \% Noise & \multicolumn{3}{|c|}{ ATMED } & \multicolumn{3}{c|}{ ATMAV } & \multicolumn{3}{c|}{ FSMF } & \multicolumn{3}{c|}{ MCFF } \\
\cline { 2 - 14 } & Im.1 & Im.2 & Im.3 & Im.1 & Im.2 & Im.3 & Im.1 & Im.2 & Im.3 & Im.1 & Im.2 & Im.3 \\
\hline 10 & 29.9 & 22.1 & 25.4 & 27.1 & 21.3 & 24.8 & 30.03 & 23.0 & 25.6 & 20.3 & 22.3 & 24.7 \\
\hline 20 & 28.5 & 19.4 & 24.9 & 27.5 & 19.6 & 24.6 & 28.72 & 20.3 & 25.3 & 22.5 & 21.4 & 24.4 \\
\hline 30 & 26.1 & 17.3 & 23.8 & 26.8 & 17.9 & 24.3 & 26.85 & 18.8 & 23.6 & 24.1 & 20.7 & 24.4 \\
\hline 40 & 23.2 & 15.5 & 21.9 & 25.1 & 16.2 & 23.5 & 25.86 & 16.8 & 21.1 & 24.8 & 20.3 & 24.3 \\
\hline 50 & 20.2 & 13.9 & 19.7 & 22.4 & 14.7 & 21.9 & 22.45 & 14.5 & 19.2 & 24.9 & 19.7 & 23.6 \\
\hline 60 & 17.6 & 12.5 & 17.6 & 19.6 & 12.9 & 19.9 & 19.27 & 13.6 & 17.4 & 22.1 & 18.4 & 21.2 \\
\hline 70 & 15.4 & 10.7 & 15.6 & 16.9 & 11.4 & 17.7 & 16.82 & 11.0 & 15.3 & 19.9 & 16.5 & 17.8 \\
\hline 80 & 13.7 & 9.3 & 14.1 & 15.1 & 9.9 & 15.7 & 15.10 & 9.6 & 14.5 & 13.4 & 13.7 & 13.5 \\
\hline 90 & 12.1 & 8.2 & 12.7 & 13.1 & 8.7 & 13.9 & 12.96 & 8.5 & 12.8 & 9.0 & 10.5 & 9.2 \\
\hline
\end{tabular}

In the above Table (3), Im. 1 is Lena (Color) image; Im.2 is C.T .Head image; Im.3 is Baboon (Color) image.

The PSNR results presented in Table(3) obtained with ATMED and ATMAV filters for standard Lena (color)and Baboon (color) image corrupted with salt and pepper impulsive noise are quite encouraging in the range of noise densities 10-40\% compared to those obtained with the proposed filters. However the PSNR results obtained with the proposed algorithm-II, a multi-pass cascaded fuzzy filter
(MCFF) are much encouraging at higher range of noise densities (50-90\%). For medical images containing high value details such as edges and fine lines, the performance of the proposed filter, PA-II is highly encouraging in terms of PSNR values with salt and pepper impulsive noise density up to $90 \%$.

Table 4.PSNR results obtained for basic fuzzy and the proposed fuzzy filters for different images contaminated with zero mean Gaussian noise at different noise variance (N.V).

\begin{tabular}{|c|c|c|c|c|c|c|c|c|c|c|c|c|c|}
\hline \multirow{2}{*}{$\%$ N.V. } & \multicolumn{3}{|c|}{ ATMED } & \multicolumn{3}{c|}{ ATMAV } & \multicolumn{3}{c|}{ FSMF } & \multicolumn{3}{c|}{ MPCFF } \\
\cline { 2 - 13 } & Im.1 & Im.2 & Im.3 & Im.1 & Im.2 & Im.3 & Im.1 & Im.2 & Im.3 & Im.1 & Im.2 & Im.3 \\
\hline 0.01 & 26.9 & 23.1 & 24.1 & 27.5 & 21.5 & 24.2 & 27.7 & 21.4 & 24.3 & 27.1 & 21.8 & 24.3 \\
\hline 0.02 & 24.7 & 21.5 & 22.8 & 26.1 & 20.7 & 23.4 & 26.2 & 20.5 & 23.6 & 24.9 & 20.1 & 22.1 \\
\hline 0.03 & 23.3 & 20.4 & 21.8 & 24.9 & 19.9 & 22.8 & 25.3 & 19.5 & 23.0 & 23.4 & 19.1 & 21.9 \\
\hline 0.04 & 22.2 & 19.5 & 21.0 & 24.0 & 19.4 & 22.3 & 24.8 & 19.0 & 22.7 & 22.4 & 18.1 & 21.2 \\
\hline 0.05 & 21.3 & 18.9 & 20.4 & 23.2 & 18.8 & 21.8 & 23.6 & 18.8 & 22.3 & 21.4 & 17.3 & 20.5 \\
\hline 0.06 & 20.7 & 18.3 & 19.8 & 22.7 & 18.4 & 21.3 & 23.1 & 18.5 & 21.8 & 20.8 & 16.7 & 20.0 \\
\hline 0.07 & 20.1 & 17.8 & 19.4 & 22.1 & 17.9 & 21.0 & 22.6 & 18.0 & 21.2 & 20.2 & 16.1 & 19.5 \\
\hline 0.08 & 19.7 & 17.3 & 19.0 & 21.7 & 17.5 & 20.5 & 21.8 & 17.7 & 20.7 & 19.7 & 15.7 & 19.1 \\
\hline 0.09 & 19.2 & 16.9 & 18.7 & 21.2 & 17.2 & 20.3 & 22.9 & 17.4 & 20.2 & 19.2 & 15.3 & 18.8 \\
\hline
\end{tabular}

In the above Table (3), Im.1 is Lena (Color) image; Im.2 is C.T .Head image; Im.3 is Baboon (Color) image. 

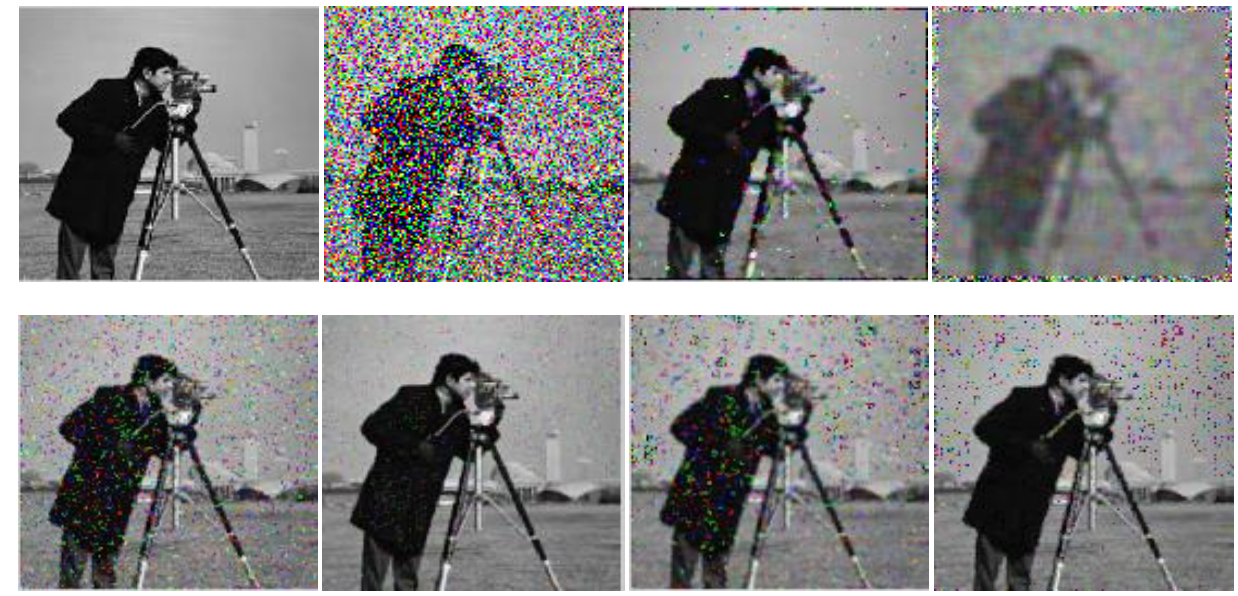

\begin{tabular}{|l|l|l|l|}
\hline $\mathbf{a}$ & $\mathbf{b}$ & $\mathbf{c}$ & $\mathbf{d}$ \\
\hline $\mathbf{e}$ & $\mathbf{f}$ & $\mathbf{g}$ & $\mathbf{h}$ \\
\hline
\end{tabular}

Fig 5:De-noising results of cameraman image with 50\% Salt and Pepper Noise (SPN). (a) Original (b) Noisy image (c) MED (d) MAV(e)ATMED (f)ATMAV(g) Fuzzy Switching Median Filter, FSMF (PA-I (h) Multi-pass Cascaded Filter, MCF (PA-II).

From the results presented in Table (4), it can be observed that, the de-noising results obtained with ATMAV and Fuzzy Switching Median Filter, FSMF (PA-I) for standard Lena (Grey) and Baboon (Color) image are much encouraging in the range of Gaussian noise with zero mean and variances 0.01-0.06 compared to those obtained with the ATMED.
However the PSNR results obtained with PA-II, a multi-pass cascaded fuzzy filter are less encouraging at higher range of noise with zero mean and variances $0.07-0.09$.For images containing rich information such as C.T. Head image (medical image), the de-noising performance of the proposed filter, PA-II is less encouraging in terms of PSNR values.

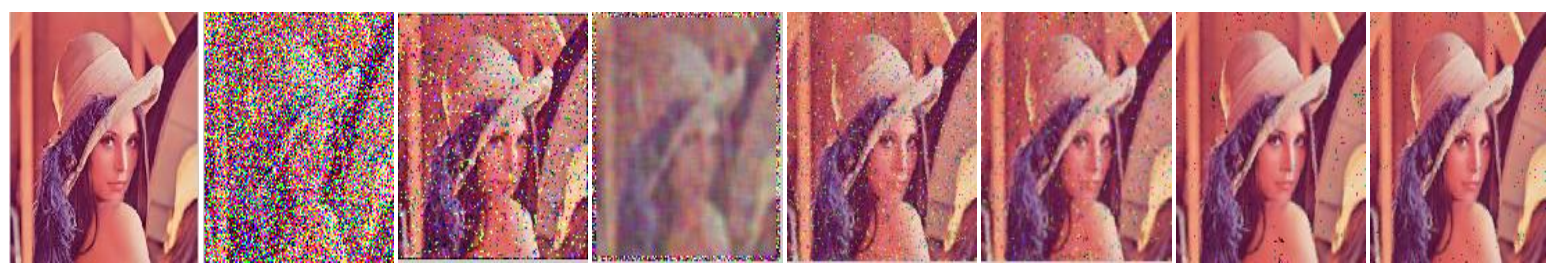

\begin{tabular}{|l|l|l|l|l|l|l|l|}
\hline $\mathbf{a}$ & $\mathbf{b}$ & $\mathbf{c}$ & $\mathbf{d}$ & $\mathbf{e}$ & $\mathbf{f}$ & $\mathbf{g}$ & $\mathbf{h}$ \\
\hline
\end{tabular}

Fig 6:De-noising results of Lena (color) image with 50\% Salt and Pepper Noise (SPN). (a) Original (b) Noisy image (c)MED (d) MAV(e)ATMED(f) ATMAV(g) Fuzzy Switching Median Filter, FSMF (PA-I) (h) Multi-pass Cascaded Filter, MCF (PA-II).
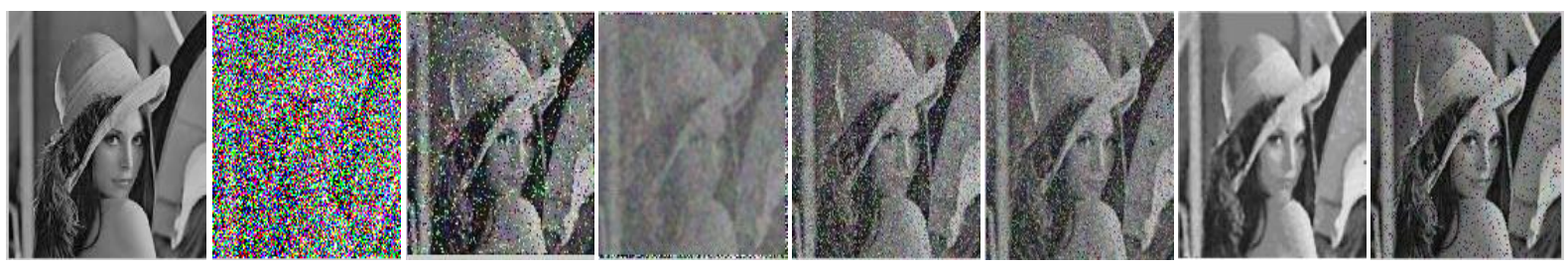

Fig 7:De-noising results of Lena (Grey, 512x512) image with 70\% Salt and Pepper Noise (SPN). (a) Original (b) Noisy image (c) MED (d) MAV (e) ATMED (f) ATMAV (g) Fuzzy Switching Median Filter, FSMF (PA-I) (h) Multi-pass Cascaded Filter, MCF (PA-II). (e) Proposed FSMF method 

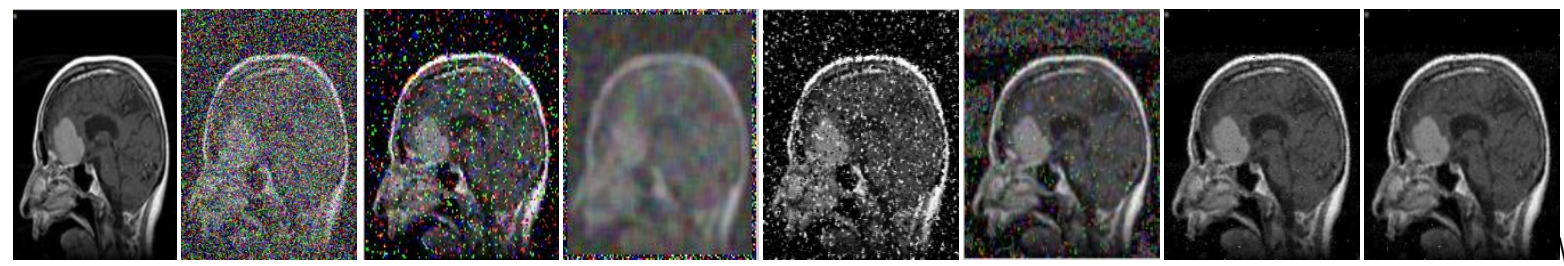

\begin{tabular}{|l|l|l|l|l|l|l|l|}
\hline $\mathbf{a}$ & $\mathbf{b}$ & $\mathbf{c}$ & $\mathbf{d}$ & $\mathbf{e}$ & $\mathbf{f}$ & $\mathbf{g}$ & $\mathbf{h}$ \\
\hline
\end{tabular}

Fig 8:De-noising results of Brain Tumor image with 50\% Salt and Pepper Noise (SPN). (a) Original (b) Noisy image (c) MED

(d) MAV(e) ATMED(f)ATMAV(g) Fuzzy Switching Median Filter, FSMF (PA-I)(h) Multi-pass Cascaded Filter, MCF (PA-II).
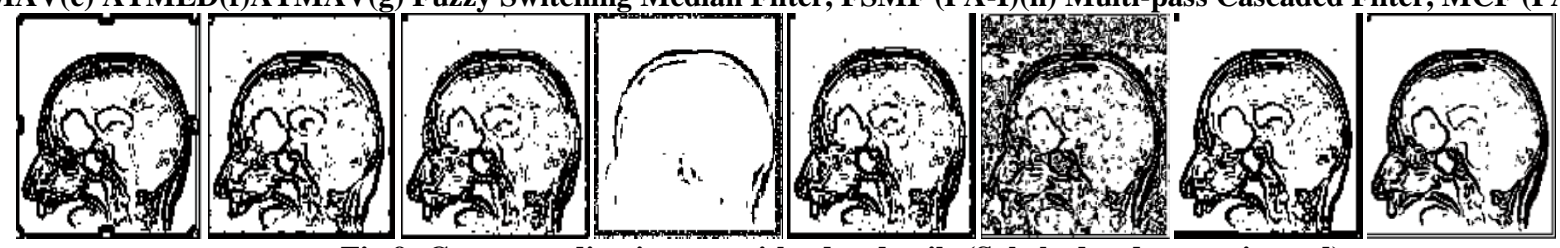

Fig 9: Corresponding images with edge details (Sobel edge detector is used).
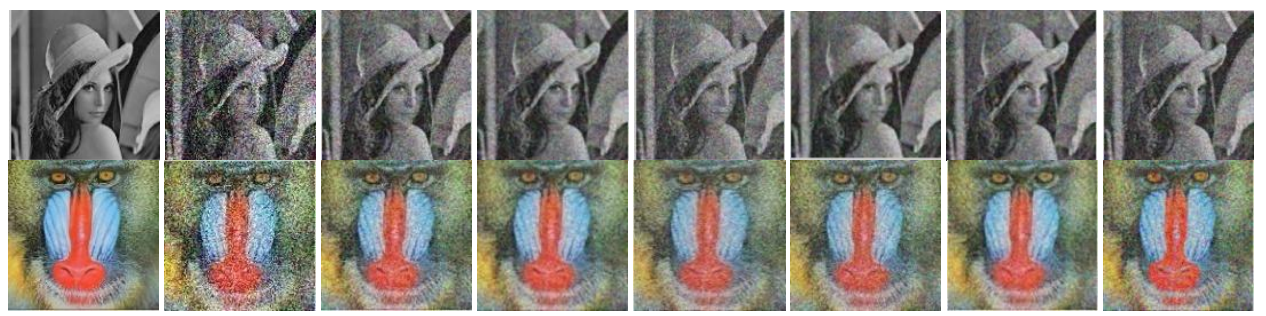

Fig 10:De-noising results of affected Lena (Grey),Baboon(color)and Gray image for zero mean Gaussian noise at noise variance 0.07 (a)Original (b) Noised (0.07) (c)MED (d) MAV (e) ATMED (f) ATMAV (g) Fuzzy Switching Median Filter, FSMF (PA-I) (h) Multi-pass Cascaded Filter, MCF (PA-II).

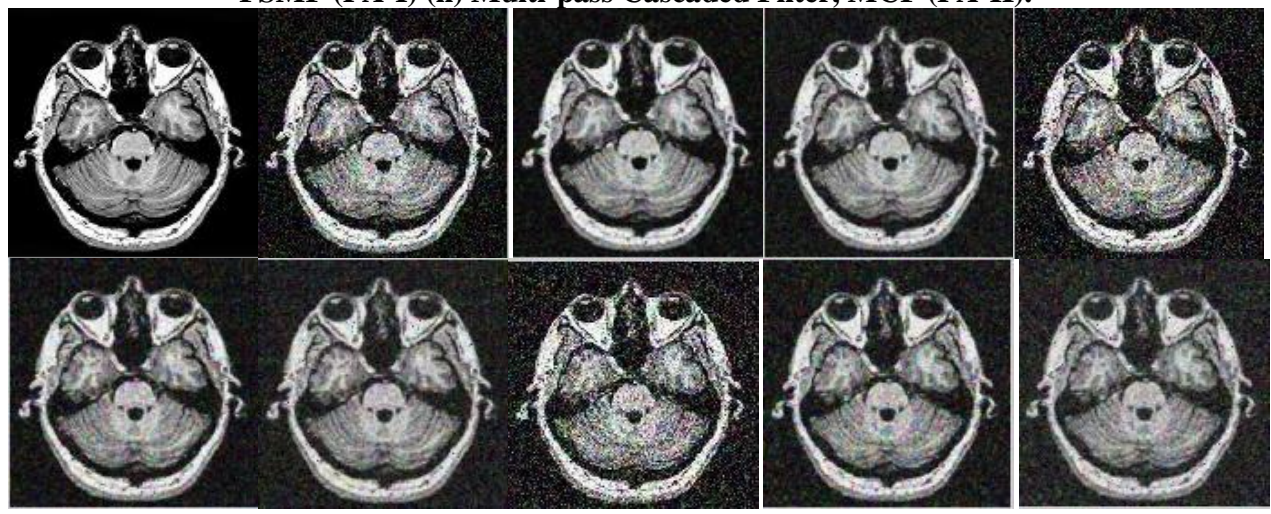

\section{\begin{tabular}{|l|l|l|l|l|}
\hline $\mathbf{a}$ & $\mathbf{b}$ & $\mathbf{c}$ & $\mathbf{d}$ & $\mathbf{e}$ \\
\hline $\mathbf{f}$ & $\mathbf{g}$ & $\mathbf{h}$ & $\mathbf{i}$ & $\mathbf{j}$ \\
\hline
\end{tabular}}

Fig 11:De-noising results of affected CT Head image for zero mean Gaussian noise at variance $0.03,0.05,0.07$ obtained for PAI and PA-II. (a)Original (b) Noised (0.03) (c)PA-I (d) PA-II (e) Noised (0.05) (f) PA-I (g)PA-II.(h) Noised 0.07 (i)PA-I (j)PA-II.

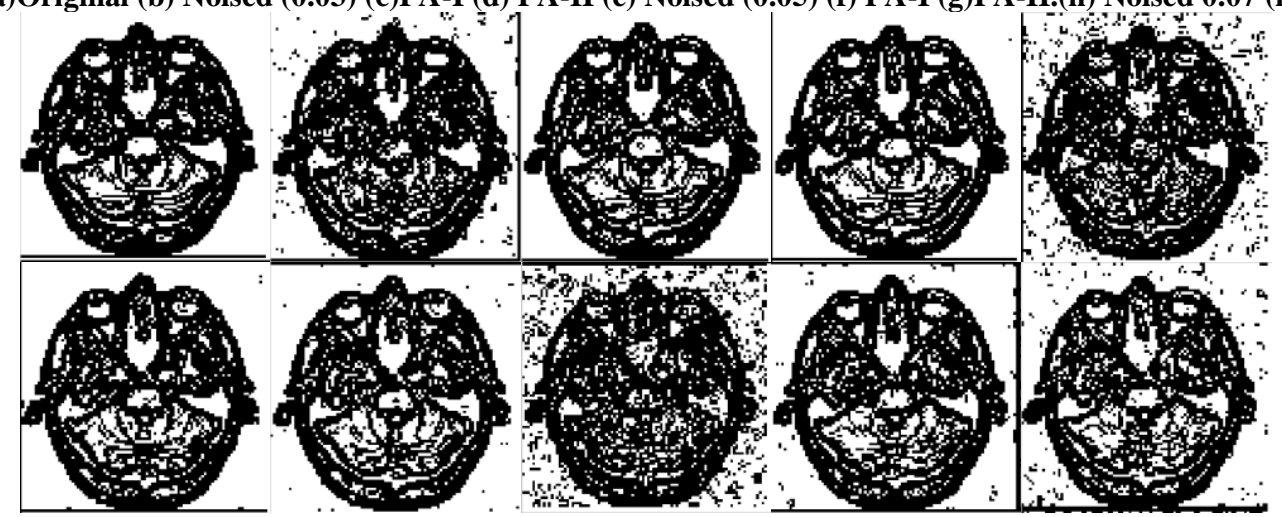

Fig 12: Corresponding images with edge details (Sobel edge detector is used). 
Visual de-noising results presented in Figures(4-12) suggest that both the proposed filters perform quite satisfactorily in de-noising grey as well as color images such as Lena, Baboon (MATLAB data base images) and clinical images such as Brain Tumor, C.T. Head images in comparison with the basic median filter, classical fuzzy median filters . Comparing the de-noising performance of the PA-I with that of proposed filter-II, it can be observed that the perceptual quality of the de-noised images obtained with proposal-II are much better for the case of medical images corrupted with impulsive and Gaussian noise of different compositions. However the PSNR results of the proposal-II are (almost) not in accordance with the visual results because, the proposed filter-II is an iterative and cascaded filter.

\section{CONCLUSIONS AND FUTURE SCOPE}

Work presented through this paper aimed at studying the classical and fuzzy classical filters and proposing; (i) a switching ATMED filter, which is a two-stage extension of the basic ATMED filter and(ii) A multi-pass cascaded for denoising images contaminated with impulsive noise at higher noise densities and Gaussian noise at moderate noise variances satisfactorily. From the results presented in the form of tables, graphs and the perceptions, the following broad conclusions can be drawn.

i) ATMED performs best in de-noising medical images affected with Gaussian noise over a wide range of noise variance.

ii) De-noising performance of the proposed filter-II, MCFF is quite satisfactory in de-noising the medical images compared to proposed algorithm-I, FSMF and other filters over a wide range of zero mean Gaussian noise at different values of noise variance.

iii)In general, the de-noising performance of ATMED, ATMAV and the proposed variants are much encouraging in de-noising Gaussian noise affected images compared to fixed value impulse noise (or SPN) affected images over a wide range of noise.

iv)These results suggest that, the basic fuzzy filters and their enhanced/modified versions are quite satisfactory in denoising images contaminated with Gaussian noise compared to salt and pepper impulsive noise (SPN).

v) Another general conclusion is that, the computed PSNR (and MSE) results and the perceived image quality are not in accordance with each other for the proposed fuzzy filters. That is, though the PSNR results of de-nosed images are high, the aesthetics and the information contents of the de-noised images are not quite acceptable. In general it can be concluded that the proposed filters, are capable performing much better in de-noising images with moderate amounts of details such as Lena (Grey) affected with Gaussian noise zero mean and variance in the range 0.01-0.09.
Further work may look at suggesting the better modifications/enhancements by the researchers in providing much better results for de-noising images affected with fixed value impulsive noise and Gaussian noise and also consider the de noising of random valued impulsive noise affected images.

\section{REFERENCES}

[1] Gonzalez, R. C. and Woods, R. E. 2002. Digital Image Processing, Second Edition, Prentice Hall, USA.

[2] Russo, F. 1998, Recent Advances in Fuzzy Techniques for Image Enhancement. IEEE Transaction and Measurement, 47(6), pp: 1428-1434.

[3] Tukey J.W.: Exploratory Data Analysis Reading, Addison Wesley.in, (1971).

[4] Bovik A.C.: Hand book of Image and Video Processing, Academic Press, (2000).

[5] Kwan. H. K. and Cai. Y. 1993, Median Filtering Using Fuzzy Concept. Proceeding of 36th Midwest Symposium on Circuits and Systems, Detroit, USA, 2, pp: 824-827.

[6] Kwan, H. K. and Cai, Y. 2002, Fuzzy Filters for Noisy Image Filtering. IEEE Transaction on Image Processing, 16(5), pp: 152-164.

[7] V.Gouda, Geeta. Hanji, Vijay Katage, M.V.Latte,2010, Impulse Noise Removal from Highly Corrupted Images", International conference on Communication, computation, control and Nanotechnology, ICN-2010,2930, Oct 2010, organized by REC, Bhalki, Bidar district, Karnataka, India.

[8] Nachtegael, M., Van Der Weken, D., Van De Ville, A. Kerre, E., Philips, W. and Lemahieu, I. 2001.An Overview of Classical and Fuzzy-Classical Filters. Proceedings of IEEE International Conference of Fuzzy Systems, pp: 3-6.

[9] Gnanamballlango and Marudhachalam. R., 2011, "New Hybrid Filtering Techniques for Removal of Gaussian Noise From Medical Images", ARPN Journal of Engineering and Applied Sciences, Vol. 6, No.2, 8-12.

[10] Marudhachalam. R., and Gnanamballlango., 2011, "Center Weighted Hybrid Filtering Techniques for Denoising of Medical Images", Proceedings of the World Congress on Engineering and Technology (CET2011), Vol. 2, 542-545.

[11] Marudhachalam. R., and Gnanamballlango., 2012, "Fuzzy Hybrid Filtering Techniques for Removal of Random Noise from Medical Images", Int. Journal of Computer Applications, Vol. 38, No. 1, 15-18.

[12] K. Arojawa, "Median Filter based on Fuzzy Rules and its Application to Image Restoration", Fuzzy Sets and Systems, Vol. 77, pp. 3-13, 1996.

[13] K. Arojawa, Edited by E.E. Kerre and M. Nachtegael, 2000, "Fuzzy Ruled-Based Image Processing with Optimization”, Springer-Verlag, pp. 222-247. 